\title{
Consideraciones legales sobre las notas de evolución en odontología.
}

\author{
Legal considerations on medical charts in dentistry.
}

\author{
Agustín Tiol-Carrillo*
}

\section{RESUMEN}

El expediente clínico es considerado un documento de importancia médica y legal en donde se integran los datos necesarios para registrar el diagnóstico y los tratamientos realizados en cada paciente. Uno de los elementos más importantes dentro del expediente clínico son las notas de evolución, documentos con los que el odontólogo informa sobre el estado general del paciente y los tratamientos realizados cita tras cita. Existen legislaciones específicas en México que orientan al estomatólogo sobre los componentes mínimos necesarios que una nota de evolución debe tener; sin embargo, una de las omisiones más comunes de los odontólogos es que, por desconocimiento, no se dé la debida importancia a la elaboración de una adecuada nota de evolución, aumentando el riesgo de problemas legales. El objetivo del presente artículo es analizar la importancia de las notas de evolución dentro del expediente clínico, destacando su importancia clínica y legal.

Palabras clave: Expediente clínico, documentación médica, nota de evolución, odontología legal.
ABSTRACT

The clinical file is considered a document of medical and legal importance where the data necessary to record the diagnosis and the treatments performed on each patient are integrated. One of the most important elements within the clinical records are the medical charts, documents through which de dentist reports on the general condition of the patient and the treatments performed appointment after appointment. There are specific laws in Mexico that guide the stomatologist on the minimum necessary components that a medical chart must have, however, one of the most common omissions of dentist is that, due to ignorance, due importance is not given to the preparation of an adequate medical chart, increasing the risk of legal problems. The aim of this article is to analyze the importance of the evolution charts within the clinical records, highlighting their clinical and legal importance.

Keywords: Clinical record, medical documentation, medical charts, legal odontology.

\section{INTRODUCCIÓN}

$\mathrm{E}$ I expediente clínico es un elemento ineludible en la Latención clínica de los pacientes que acuden a consulta, ya que en él se plasman todos los registros organizados del proceso salud-enfermedad que permitan constatar la buena práctica médica a través de la elaboración de una buena historia clínica, una adecuada exploración física, el archivamiento de fotografías y radiografías, y un conveniente llenado de las notas de evolución por cada procedimiento realizado.

Las notas de evolución son una herramienta con la que los profesionales de la salud registran el historial médico del paciente y que, a su vez, permiten comprender el diagnóstico dado, así como el continuo registro de los tratamientos realizados y las necesidades de cada paciente, ${ }^{1}$ por tanto, la importancia de las notas de evolución radica en que en ellas no sólo se registre el conjunto de signos y

\footnotetext{
* Especialista en Estomatología Pediátrica. Máster en Odontología Legal y Forense. Profesor investigador de tiempo completo en la UAMXochimilco. México.

Recibido: 10 de julio de 2021. Aceptado: 12 de septiembre de 2021.

Citar como: Tiol-Carrillo A. Consideraciones legales sobre las notas de evolución en odontología. Rev ADM. 2021; 78 (5): 280-282. https://dx.doi. org/10.35366/102036
} 
síntomas del padecimiento que afectan al paciente, sino que también se reporte el seguimiento que el profesional sanitario ha dado al padecimiento..$^{2,3}$

Todos los procedimientos odontológicos tienen un conjunto de métodos para poderlos ejecutar de forma conveniente; sin embargo, un mismo procedimiento jamás será igual en todos los pacientes, ya que cada intervención presenta siempre específicas peculiaridades en virtud del estado actual del caso, el grado de avance del padecimiento y las condiciones en las cuales se realiza un determinado procedimiento. Dichas peculiaridades deben ser registradas de la forma más completa y detallada posible a través de las notas de evolución.

El presente artículo tiene como objetivo profundizar en el trasfondo legal de las notas de evolución, destacando su importancia jurídica, tanto para la seguridad del paciente como en la protección legal del odontólogo.

\section{MARCO JURÍDICO DE LAS NOTAS DE EVOLUCIÓN EN MÉXICO}

La Ley General de Salud, en su artículo 77 Bis 37 especifica los derechos y obligaciones de todo paciente que acude a algún consultorio u hospital a recibir atención médica, dentro de los cuales destaca el contar con un expediente clínico. ${ }^{4}$

En el numeral 6 al 6.2. de la NOM-004-SSA3-2012 Del expediente clínico se especifica que el expediente clínico de consulta general y de especialidad debe contar con una historia clínica, interrogatorio, exploración física, resultados de laboratorio, diagnóstico o problemas clínicos, pronóstico, indicaciones terapéuticas y notas de evolución. ${ }^{5}$

Las notas de evolución son un componente inherente al expediente clínico, y tanto la NOM-004-SSA3-2012 como la NOM-013-SSA2-2015 concuerdan en que las notas de evolución deben elaborarse cada vez que se proporcione atención al paciente. Sin embargo, al haber dos Normas Oficiales Mexicanas con diferentes enfoques sobre el expediente clínico, se generan frecuentemente dudas en el estomatólogo, por lo que vale la pena destacar que el numeral 5.15 de la NOM-004-SSA3-2012 Del expediente clínico especifica que el expediente odontológico no debe elaborarse con las especificaciones de la NOM-004-SSA3-2012, sino que debe estructurarse apegándose en su totalidad a la NOM-013-SSA2-2015 Para la prevención y control de enfermedades bucales. ${ }^{5}$

Jurídicamente no existe un formato obligatorio para poder elaborar una nota de evolución, por tanto, ésta puede ser redactada a voluntad del clínico. No obstante, independientemente de la forma en la que se redacte, ésta debe contener elementos obligatorios en su estructura, claramente descritos del numeral 9.3 al 9.3.7 de la NOM-013-SSA2-2015, como la fecha en la que se realizó el procedimiento, la actividad realizada de la forma más explícita posible, la evolución y actualización del cuadro clínico, los signos vitales tomados en cada cita, registrar el diagnóstico, pronóstico y plan de tratamiento. Además, en caso de contar con radiografías o estudios de laboratorio, la interpretación de éstos debe estar descrita de manera minuciosa en la nota de evolución, por último, registrar a detalle el tratamiento realizado, las indicaciones terapéuticas y estomatológicas a seguir, sin olvidar los fármacos utilizados durante la consulta y posteriores a ésta mencionando la presentación, dosis, vía de administración, periodicidad y duración de los mismos, así como los posibles efectos adversos que pudieran presentarse. ${ }^{6}$

Independientemente de si la nota de evolución es redactada por un médico o un estomatólogo, ésta debe elaborarse siempre con terminología técnica, sin obviar ni omitir ningún tipo de información, absteniéndose en todo momento del uso de abreviaturas o palabras ilegibles. ${ }^{2,7}$

Toda nota de evolución deberá contener el nombre y firma del estomatólogo que la elabora, así como el nombre y la firma del paciente o su representante legal según lo especificado en el numeral 9.3.7. de la NOM013-SSA2-2015. ${ }^{6}$

\section{ESTRUCTURA DE UNA NOTA DE EVOLUCIÓN}

Como ya se mencionó con antelación, a pesar de que en nuestra legislación se indica la información mínima necesaria que debe contener una nota de evolución, no existe un formato específico para su elaboración.

El sistema SOAP es una excelente herramienta para elaborar una nota de evolución de la forma más completa y ordenada posible. Dicho sistema recibe este nombre por sus iniciales en inglés Subjective, Objective, Assessment y Plans, aunque se ha creado una adecuación al idioma español: Subjetivo, Objetivo, Apreciación y Planes. ${ }^{8}$

En este sistema SOAP, en los datos subjetivos se debe registrar toda aquella información proporcionada por el propio paciente y puede ser registrada con lenguaje no técnico, es decir, tal y como lo expresa; mientras que en los datos objetivos debe plasmarse toda aquella información obtenida a través de una exhaustiva exploración clínica por medio de la inspección, percusión, auscultación y palpación, así como de los resultados de 
los exámenes auxiliares realizados. La apreciación se refiere a la evaluación y comentarios que surjan como resultado de los datos obtenidos, es aquí donde el clínico expresará su opinión frente al padecimiento, ofreciendo un diagnóstico y posibles diagnósticos diferenciales. Por último, en la sección de planes se refieren las nuevas acciones a realizar tras amalgamar lo subjetivo con lo objetivo, ${ }^{9}$ es en esta sección donde el odontólogo deberá esclarecer si realizará exámenes o análisis adicionales y los tratamientos a realizar para dar solución a la problemática del paciente.

\section{LAS NOTAS DE EVOLUCIÓN EN ODONTOLOGÍA}

Una nota de evolución es una información narrativa que proporciona información sistemática y cronológica sobre la evolución del proceso de salud-enfermedad del caso que se encuentra en tratamiento odontológico. ${ }^{8,10}$ Su importancia radica en que en ella se registran todos los tratamientos realizados en cada cita. Muchos de los tratamientos odontológicos no se culminan en una sola sesión, y ciertas peculiaridades deben ser plasmadas por escrito para evitar que el odontólogo olvide información sensible que pudiera producir errores en las citas subsecuentes; ejemplo de esto podría ser la conductometría de la raíz de un diente durante un tratamiento endodóntico, la selección del color para la elaboración de prótesis fija o removible o las indicaciones postoperatorias dadas al paciente después de un tratamiento.

Toda nota de evolución debe redactarse con tal claridad y detalle que ofrezca una absoluta comprensión a cualquier clínico que la lea sobre el estado de salud inicial y final del paciente, así como los tratamientos realizados cita por cita.

Asimismo, las notas de evolución sirven como evidencia de una buena práctica médica frente a problemas de índole jurídico en contra del odontólogo, ya que permiten a la autoridad competente informarse sobre las características de los tratamientos realizados, para así poder crear o extinguir responsabilidades de orden penal o civil. ${ }^{11}$

Resulta interesante notar que, a pesar de la gran importancia que tienen las notas de evolución tanto en la protección y seguridad del paciente como del propio odontólogo, sean elementos comúnmente omitidos o mal elaborados por los mismos profesionales por considerarlas poco importantes o como una pérdida de tiempo, sin detenerse a pensar que, ante problemas legales, las notas de evolución serán determinantes en la toma de decisiones de los peritos y jueces.

\section{CONCLUSIÓN}

Como se habrá visto a lo largo del presente artículo, las notas de evolución son componentes inherentes al expediente clínico que, de acuerdo con nuestra legislación, deben ser redactadas cada vez que el odontólogo preste atención a cualquier paciente.

El contar con un buen expediente clínico con notas de evolución completas y adecuadamente redactadas es un claro ejemplo de una buena práctica clínica que se inculca fuertemente en la formación profesional de todo odontólogo a nivel universitario, y resulta abrumador notar que numerosos odontólogos abandonen de manera voluntaria las buenas prácticas, omitiendo la elaboración del expediente clínico y los registros de las actividades realizadas, aumentando notablemente el riesgo de sufrir problemas legales. Cabe destacar que toda vez que un odontólogo decida brindar atención a un paciente sin haber realizado un expediente clínico completo y sin notas de evolución, está actuando con negligencia.

\section{REFERENCIAS}

1. Suti Ismawati ND, Supriyanto S, Haksama S, Hadi C. The influence of knowledge and perceptions of doctors on the quality of medical records. J Public Health Res. 2021; 10 (2): 2228.

2. Carmona-Mejía B, Ponce-Gómez G. Evaluación del expediente clínico integrado y de calidad en pacientes de cirugía programada. Rev CONAMED. 2014; 19 (3): 117-127.

3. Ramírez RA. Introducción al tema de la documentación del acto médico. Rev CONAMED. 2007; 12 (1): 29-30.

4. Ley General de Salud. 2021.

5. NOM-004-SSA3-2012 Del expediente clínico. Diario Oficial de la Federación.

6. NOM-013-SSA2-2015 Para la prevención y control de enfermedades bucales. Diario Oficial de la Federación.

7. Reyna-Lara M. Derechos y obligaciones de los usuarios del servicio de salud. Med Int Mex. 2018; 34 (5): 780-791.

8. Sando KR, Skoy E, Bradley C, Frenzel J, Kirwin J, Urteaga E. Assessment of SOAP note evaluation tools in colleges and schools of pharmacy. Curr Pharm Teach Learn. 2017; 9 (4): 576-584.

9. Muñoz J. El registro medico orientado por problemas. An Fac Med. 1998; 59 (1): 73-78.

10. Medrano MJ. Expediente clínico odontológico. Cómo elaborarlo, integrarlo, manejarlo y archivarlo conforme a la normatividad. México: Trillas; 2018. pp. 45-46.

11. Tiol-Carrillo A. El peritaje odontológico forense. Rev Odont Mex. 2016; 20 (3): 154-158.

Conflicto de intereses: El autor declara no tener ningún conflicto de intereses.

Financiamiento: Ninguno.

Correspondencia:

Agustín Tiol-Carrillo

E-mail: agustintiolcarrillo@gmail.com 